\title{
How to prevent inadvertent emergency anticoagulation in acute type A aortic dissection: when in doubt, don't
}

\author{
Silke Zschaler ${ }^{1}$, Gerard Schmidt ${ }^{2}$, Marian Kukucka ${ }^{1}$, Georg Syrmas ${ }^{3}$, Lisa Zaschke ${ }^{1}$, Stephan Dominik Kurz ${ }^{1}$ \\ ${ }^{1}$ Institute for Anesthesiology, ${ }^{2}$ Department of Cardiothoracic and Vascular Surgery, Deutsches Herzzentrum Berlin, Berlin, Germany; ${ }^{3}$ Department \\ of Emergency Medicine, Campus Benjamin Franklin (CBF), Charité-Universitätsmedizin Berlin, Berlin, Germany \\ Correspondence to: Dr. rer. medic. Stephan Dominik Kurz, MPH. Institute for Anesthesiology, Deutsches Herzzentrum Berlin, Augustenburger Platz 1, \\ 13353 Berlin, Germany. Email: skurz@dhzb.de.
}

\begin{abstract}
Inadvertent emergency anticoagulation in patients with acute type A aortic dissection (ATAAD) has been sparsely reported. There are case reports bringing this potential critical incident to attention, however, little is known about the number of undetected and unreported cases. We approach this issue based on a case report of inadvertent emergency anticoagulation in ATAAD and attempt to shed light on aspects that may have contributed to the critical incident: The challenge of distinguishing an ATAAD from an acute coronary syndrome (ACS) and the potential underestimation of incidents of ATAAD. We also discuss errors and biases in medical decision making, and provide suggestions that may help raise awareness of how ATAAD can be mimicking ACS in clinical practice.
\end{abstract}

Keywords: Aneurysm; dissecting; anticoagulants; aortic rupture; emergency medical services; patient safety

Submitted Jul 31, 2018. Accepted for publication Oct 16, 2018.

doi: $10.21037 / \mathrm{cdt} .2018 .10 .13$

View this article at: http://dx.doi.org/10.21037/cdt.2018.10.13

Inadvertent emergency anticoagulation in patients with acute type A aortic dissection (ATAAD) has been sparsely reported since von Kodolitsch et al. (in 2000) first addressed this problem in their work (1). There are numerous case reports bringing this potential critical incident to attention, either by pointing out the devastating consequences on a theoretical basis, or by reporting cases where fortunately no antiplatelet, anticoagulation or antithrombotic therapy had been administered $(2,3)$. In contrast to early anticoagulation, late anticoagulation after surviving ATAAD has been found to not impact late mortality (4).

In the first part of the article, we briefly report a case of inadvertent emergency anticoagulation. In the second part, we discuss aspects that may lead to misdiagnosing ATAAD as acute coronary syndrome (ACS) and discuss the possible reasons contributing to us not taking ATAAD into account. In the last part, we suggest a practical approach that might help overcoming cognitive dispositions to respond (CDR) to a patient presenting with chest pain.

\section{A case of inadvertent anticoagulation in ATAAD}

Emergency medical services had been called to an awake, oriented and coherent 64-year-old man who suffered an abrupt onset of chest pain. He was an active cigarette smoker (20 cigarettes per day, unknown number of pack years). Aside from morbid central obesity and arterial hypertension, his medical history was empty.

The physical examination revealed clinical signs consistent with malperfusion of the right leg. If the aortic dissection detection risk score (ADD-RS) had been used, the patient would have scored 2. With an ADD-RS of 2, the patient would have been triaged as 'high risk' requiring immediate aortic imaging and referral to a facility capable of operative management of ATAAD (5).

The electrocardiogram (ECG) showed depression of the ST-segment in V4-V6 and ST elevation in aVR and V1. The emergency physician started a combination of a dual antiplatelet therapy with acetylsalicylic acid (ASA) 
and clopidogrel and an intravenous anticoagulation with unfractionated heparin. The patient was dispatched to the chest pain unit (CPU) at an urban tertiary hospital.

$\mathrm{He}$ arrived at the CPU with a Glasgow Coma Score of 15. High-sensitive cardiac troponin $\mathrm{T}$ turned out positive. In the emergency coronary arteriography of the referring hospital, the coronary arteries were not visible; and the subsequent computed tomography scan of the chest revealed an ATAAD. No further radiographic information was provided by the referring hospital.

The patient was dispatched to the Deutsches Herzzentrum Berlin (DHZB). Shortly before the intensive care transport arrived, he went into circulatory failure and required cardiopulmonary resuscitation. After administering epinephrine, spontaneous circulation returned after one minute. He was transferred to the DHZB sedated, intubated, ventilated and under continuous administration of norepinephrine.

He arrived at the DHZB sufficiently oxygenated and hemodynamically stable under norepinephrine. Transthoracic ultrasound showed an aortic root aneurysm and an ATAAD with the dissection membrane being connected to the semilunar valves, causing an acute aortic insufficiency grade III.

With a total "pain-to-cut-time" (PCT) of 5.5 hours, the patient received a total aortic root replacement with a biological valved conduit and a reconstruction of the aortic arch. Intraoperatively, he developed disseminated intravascular coagulopathy requiring a massive transfusion of 7.5 liters autologous blood from cell salvage, 20 units of red cell concentrate, 56 units of fresh frozen plasma and 11 units of platelet concentrate. He was also given $24 \mathrm{mg}$ of recombinant activated factor VII.

Postoperatively initially stable, his gas exchange became severely restricted and the postoperative hemorrhage did not subside. A sudden increase of liver and intestinal enzymes led to an emergency surgery where a bare metal stent was placed in the collapsed true lumen of the descending aorta. Continuously increasing indicators of shock and an ongoing hemodynamic instability demanded an increased administration of catecholamines. Based on a metabolic crisis, the patient began to develop cardiac arrhythmias culminating in pulseless ventricular tachycardia. Cardiopulmonary resuscitation failed despite multiple defibrillations, intermittent bolus doses of epinephrine and mechanical resuscitation 60 hours after the onset of chest pain.

The autopsy report revealed signs of severe generalized arteriosclerosis, signs of chronic obstructive pulmonary disease and signs of hypertension. A functional remnant dissection of the entire aorta originating at the graft going all the way down to the aortic bifurcation was found. There was no intimal tear or bleeding into the arterial walls. Death by protracted cardiogenic shock with consecutive multiorgan failure was confirmed.

\section{Chest pain and emergency anticoagulation}

In a pre-hospital setting, distinguishing ATAAD from ACS when the chief complaint is chest pain is challenging; it requires a high level of clinical suspicion and a meticulous clinical evaluation $(2,6)$. Chest pain with an abrupt onset can indicate pulmonary embolism, aortic dissection and pneumothorax. But in clinical practice, it is primarily linked to myocardial underperfusion $(6,7)$. Patients with ATAAD also may display ECG signs of malperfusion, but they do not necessarily develop myocardial ischemia (8).

Early antiplatelet therapy and anticoagulation in patients with ACS is the first line of therapy to antagonize the coagulation cascade and to minimize procedural risks in percutaneous coronary intervention (9). In patients with ATAAD misdiagnosed as ACS and the administration of anticoagulants can have lethal consequences.

Davis et al. (in 2004) investigated the medical records of an emergency department and identified 44 patients with non-traumatic ATAAD. Nine of them were inadvertently anticoagulated. One patient who received fibrinolytics died of bleeding complications before aortic surgery. One patient who was given heparin and ASA required a repeat thoracotomy due to bleeding complications, another patient who received ASA suffered an embolic stroke. Two of three patients who were given a combination of heparin and ASA died; one of bleeding complications before aortic surgery, one suffered a stroke after the reversal of anticoagulation (10).

Davis et al. (in 2004) reported patients were more likely to receive an inadvertent emergency anticoagulation if they presented with chest pain without back pain, and if their ECG showed depression or elevation of the ST-segment (10).

ATAAD and ACS are not mutually exclusive. There are case reports about ACS and concomitant ATAAD, and about ATAAD being a precursor to ACS $(7,11)$. ATAAD can leave the same clinical impression as ACS when the right coronary artery (RCA) (9), the left main coronary artery (LMCA) (12) or both (13) are involved. Studies suggest ATAAD might affect the RCA more than the LMCA $(13,14)$.

Another aspect needs to be taken into consideration in the broader context of inadvertent anticoagulation: What 
happens to heavily anticoagulated patients who suffer an ATAAD? Hamad et al. (in 2018) brought the increasing prevalence of non-vitamin $\mathrm{K}$ antagonist oral anticoagulants (NOACs) to attention. There is no documented consensus for the surgery management of patients with ATAAD who are taking rivaroxaban or dabigatran $(15,16)$. An appropriate delay of the emergency surgery may have to be considered to reverse the anticoagulant effect of the NOACs to keep the mortality risk within an acceptable range $(15,16)$.

\section{Underestimated incidences of ATAAD}

The current state of research classes ATAAD as a rare occurrence. Estimation rates from pre- and in-hospital studies, and population-based studies range from three to four cases per 100,000 people per year (17). A Swedish nationwide study suggested an incidence of 2.5 cases per 100,000 people per year after adjusting for age and gender (18). Data retrieved in the context of helicopter and groundbased emergency medical services are rather sparse, and potentially biased by the high pre-hospital mortality of those patients (19).

According to the literature available, the probability of being confronted with ATAAD may invite us to reminiscence the famous advice by Dr. Theodore Woodward: "When you hear hoofbeats, think of horses, not zebras." (20).

In an US-American retrospective cohort study, Alter et al. (in 2015) analyzed 9,533,827 patient contacts that took place in 33 emergency departments in both suburban and urban New York including New Jersey between 19962010. They identified 550 confirmed cases of ATAAD in 14 years (21). Based on that study, ATAAD would be classed a zebra. Referring to their calculations, the authors stated an emergency physician in a hospital setting would roughly get to diagnose an aortic dissection every 3 to 4 years if they saw 3,000 to 4,000 patients every year (21).

Our recent study considers it worthwhile thinking about ATAAD as a horse rather than a zebra. From 2010-2014, Kurz et al. (in 2017) investigated all in-hospital patients being admitted to two of the largest cardiac surgical centers in Berlin. At that time, the urban and suburban region of Berlin and Brandenburg was inhabited by minimum $5,779,182$ and maximum 5,963,988 people. The German retrospective cohort study revealed 405 confirmed cases of ATAAD in five years and identified additional 145 lethal ATAAD cases in 14,201 autopsy reports (19).

Based on these findings, the incidents of 11.9 cases per 100,000 people per year for the region of BerlinBrandenburg exceeded the incidence of 4.63 cases per 100,000 people per year the Federal Republic of Germany published for 'thoracic aortic dissection' based on the official mortality statistics (19).

Considering the time frames, these two studies are in line with findings from differently designed and sometimes hardly comparable epidemiologic studies stating an increasing prevalence up to $15-16$ cases per 100,000 inhabitants per year for thoracic aortic aneurysm and acute aortic type A dissection (22).

If we take the broad symptom spectrum of ATAAD Hagan et al. (in 2000) described into consideration, we assume the incidence of the New York study is vastly underrated.

\section{Errors and biases in (mis-)diagnosing ATAAD}

Literature identified three types of error in medical decision-making: non-fault errors, system errors and cognitive errors (23).

Non-fault errors are referring to a disease presenting in any other way that its pathognomonic appearance. It may present silent, atypically or mimic something else (24). Patients are more likely to present with a broad variety of uncommon symptoms $(23,25)$. The clinical impression may be dominated by a variety of benign medical issues, or the clinical course may be silent (26). Nadour et al. (in 2008) reported a case of silent aortic dissection presenting as transient locked-in syndrome (27).

System errors leading to delaying or missing the diagnosis are caused by imperfections in patient care (24). After reviewing a critical incident, not having access to the expert knowledge of radiologists in an emergency department has been identified as a system error contributing to misdiagnosing ATAAD (25).

Cognitive errors are common in medicine and reflect a human being's limitations to process information, therefore they are ineradicable $(24,25,28)$. How information is presented subconsciously influences the choices we make. Our human nature allows us to respond to certain situations in a variety of predictable ways (29). Croskerry (in 2003) published more than 30 of those CDR in the context of medical errors (Table 1).

The availability bias contributes to confusing ATAAD with ACS, because chest pain is inextricably linked to ACS. Anchoring and order effects become relevant when a patient with ATAAD is admitted with suspected ACS. Confirmation 
Table 1 Selected CDRs by Croskerry (in 2003) relevant to the emergency diagnostic process of chest pain (28)

CDRs that may lead to diagnostic error

Anchoring: the tendency to perceptually lock onto salient features in the patient's initial presentation too early in the diagnostic process, and failing to adjust this initial impression in the light of later information. This CDR may be severely compounded with the confirmation bias

Availability bias: the disposition to judge things as being more likely, or frequently occurring, if they readily come to mind. This, recent experience with a disease may inflate the likelihood of its being diagnosed. Conversely, if a disease has not been seen for a long time (is less available), it may be underdiagnosed

Commission bias: results from the obligation toward beneficence, in that harm to the patient can only be prevented by active intervention. It is the tendency toward action rather than inaction. It is more likely in over-confident physicians

Confirmation bias: the tendency to look for confirming evidence to support a diagnosis rather than to look for disconfirming evidence to refute it; despite the latter often being more persuasive and definite

Search satisfying: reflects the universal tendency to call off a search once something is found. Comorbidities, second foreign bodies, other fractures, and coingestants in poisoning may all be missed. Also, if a search yields nothing, diagnosticians should satisfy themselves that they have been looking in the right place

Premature closure: a powerful CDR accounting for a high proportion of missed diagnoses. It is the tendency to apply premature closure to the decision-making process, accepting a diagnosis before it has been fully verified. The consequences of the bias are reflected in the maxim: "When the diagnosis is made, the thinking stops."

Sutton's slip: takes its name from the apocryphal story of the Brooklyn bank-robber William Sutton who, when asked by the judge why he robbed banks, is alleged to have replied: "Because that's where the money is!" The diagnostic strategy of going for the obvious is referred to as Sutton's Law. The slip occurs when possibilities other than the obvious are not given sufficient consideration

Visceral bias: the influence of affective sources of error on decision-making has been widely underestimated. Visceral arousal leads to poor decisions. Countertransference, both negative and positive feelings towards patients, may result in diagnoses being missed. Some attribution phenomena (fundamental attribution error) may have their origin in countertransference

CDRs, cognitive dispositions to respond.

bias, search satisfying, Sutton's slip and premature closure are processes that may explain the phenomenon Garber et al. (in 2014) described as "an existing diagnosis has almost infinite inertia".

When debating medical decision-making in emergency medicine, we must consider patients are often highly stressed, agitated or suffering. In the background of inadvertent emergency anticoagulation, we suspect visceral bias and commission bias to be influential CDRs.

\section{Practical implementations}

Diagnostic errors can never be eliminated, but they can be reduced $(24,25,28)$. Overcoming the overlearned procedures and the automated response of anticoagulating patients presenting with chest pain is necessary to improve patient safety. Consistent use of the ADD-RS prior to the administration of anticoagulants may protect many patients from an inadvertent iatrogenic critical incident (5).

\section{Rule out the worst case}

ATAAD should always be a differential diagnosis to consider in patients with chest pain; and ruling out the worst case in pre-hospital and emergency settings is appropriate $(12,25)$.

Consider ATAAD occurs more often than epidemiologic studies may suggest. Even if you are not convinced and prefer to look at ATAAD as a rare entity, keep in mind the rarity of a condition does not alter the impact it has on the odds of an individual person (29). Focus should be on the patient you are diagnosing.

\section{Accept uncommon is common, and classic is rare}

Harvey et al. (in 1979) state: "If the diagnosis can be made on the basis of specific criteria, then these criteria are either fulfilled or not fulfilled."

The uncommon and non-specific presentation of the clinical symptoms and radiology findings defines the 


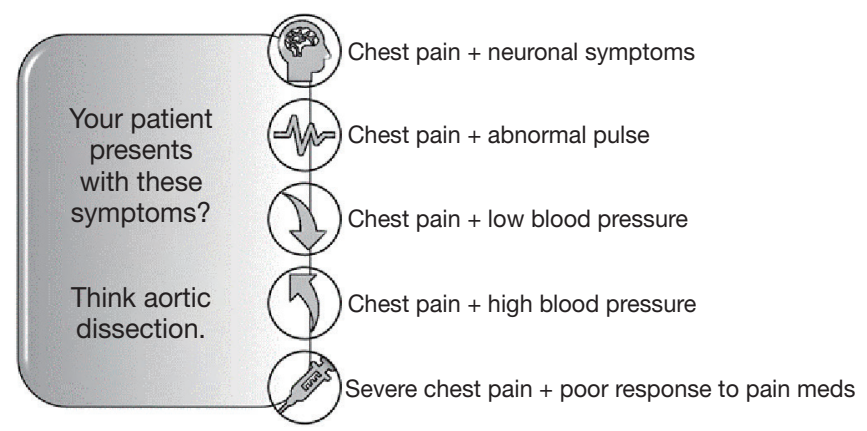

Figure 1 Symptom combinations prompting ATAAD as suggested by Gisondo (30). ATAAD, acute type A aortic dissection.

diagnostic norm (27). It is not the lack of knowledge that fails to bring ATAAD out of the focus, but the link between what we know and what we see $(25,30)$.

To increase patient safety, we suggest establishing a handful of easily memorable red flags that may help you establishing a broader view on chest pain (Figure 1).

\section{Trust your doubt}

If you find yourself confused and feel like your patient does not fit the diagnosis that springs to your conscious mind, we suggest you take a step back and allow yourself time to think.

Considering an alternative diagnosis when you are already dealing with inconsistencies might provide you with the cues you need to rethink a case: an immeasurable blood pressure and normal consciousness might not add up to severe hypotension; a sudden onset of chest pain and neurological symptoms do not meet the time criteria for embolization $(2,25)$.

\section{Keep an open mind}

If there is no doubt, rather than attempting to set your diagnosis in stone, make ruling out premature closure your priority. Garber (in 2014) suggests to routinely generate an alternative differential diagnosis in every case.

Get to know yourself better. By taking a meta-position, you will learn about the CDRs you are confronted with. It is important to understand the cognitive biases we have become accustomed to and to adapt strategies to minimize the likelihood of being trapped in them without realizing (25).

\section{Acknowledgements}

We would like to thank Tegan Alexandra Steen of Melbourne, Australia for her assistance in linguistics on this manuscript.

\section{Footnote}

Conflicts of Interest: The authors have no conflicts of interest to declare.

\section{References}

1. von Kodolitsch Y, Schwartz AG, Nienaber CA. Clinical prediciton of aortic dissection. Arch Intern Med 2000;160:2977-82.

2. Marek D, Nemec P, Herman M, et al. Mistakes in dealing with aortic dissection. Lessons from three warning cases. Biomed Pap Med Fac Univ Palacky Olomouc Czech Repub 2008;152:283-7.

3. Forbes RA, Balderston G. Aortic dissection mimicking acute myocardial infarction: the perils of prehospital care. Prehosp Emerg Care 2008;12:503-5.

4. von Kodolitsch $\mathrm{Y}$, Wilson $\mathrm{O}$, Schüler $\mathrm{H}$, et al. Warfarin anticoagulation in acute type A aortic dissection survivors (WATAS). Cardiovasc Diagn Ther 2017;7:559-71.

5. Rogers AM, Herman LK, Booher AM, et al. Sensitivity of the Aortic Dissection Detection Score, a Novel GuidelineBased Tool for Identification of Acute Aortic Dissection at Initial Presentation. Results from the International Registry of Acute Aortic Dissection. Circulation 2011;123:2213-8.

6. Erhardt L, Herlitz J, Bossaert L, et al. Task force on the management of chest pain. Eur Heart J 2002;23:1153-76.

7. Lentini S, Perrotta S. Aortic dissection with concomitant acute myocardial infarction: From diagnosis to management. J Emerg Trauma Shock 2011;4:273-8.

8. Martin JF, Andrade LG, Loureiro AA, et al. Myocardial infarction and aortic dissection: an important differential diagnosis. Rev Bras Cir Cardiovasc 2004;19:386-90.

9. Zeitouni M, Kerneis M, Nafee T, et al. Anticoagulation in Acute Coronary Syndrome-State of the Art. Prog Cardiovasc Dis 2018;60:508-13.

10. Davis DP, Grossman K, Kiggins DC, et al. The inadvertent administration of anticoagulants to ED patients ultimately diagnosed with thoracic aortic dissection. Am J Emerg Med 2005;23:439-42. 
11. Dixon M. Aortic Dissection or MI? It could be both. Emergency Med 2014;4:199.

12. Camaro C, Wouters NT, Gin MT, et al. Acute myocardial infarction with cardiogenic shock in a patient with acute aortic dissection. Am J Emerg Med 2009;27:899.e3-6.

13. Neri E, Toscano T, Papalia U, et al. Proximal aortic dissection with coronary malperfusion: presentation, management, and outcome. J Thorac Cardiovasc Surg 2001;121:552-60.

14. Spittell PC, Spittell JA Jr, Joyce JW, et al. Clinical features and differential diagnosis of aortic dissection: experience with 236 cases (1980 through 1990). Mayo Clin Proc 1993;68:642-51.

15. Hamad R, Amr G, Demers P. Delayed surgery in patients with acute type A aortic dissection who are receiving novel oral anticoagulants. J Thorac Cardiovasc Surg 2018;155:e1-4.

16. Preventza O, Bracey A. Surgery for acute type A aortic dissection on oral anticoagulants: Being the dispatcher of a 911 call. J Thorac Cardiovasc Surg 2018;155:e5-6.

17. Nienaber CA, Clough RE. Management of acute aortic dissection. Lancet 2015;385:800-11.

18. Melvinsdottir IH, Lund SH, Agnarsson BA, et al. The incidence and mortality of acute thoracic aortic dissection: results from a whole nation study. Eur J Cardiothorac Surg 2016;50:1111-7

19. Kurz SD, Falk V, Kempfert J, et al. Insight into the incidence of acute aortic dissection in the German region of Berlin and Brandenburg. Int J Cardiol 2017;241:326-9.

20. Sotos JG. Zebra Cards: An Aid to Obscure Diagnoses (3rd ed.). Mt. Vernon: Mt. Vernon Book Systems, 2006.

21. Alter SM, Eskin B, Allegra JR. Diagnosis of Aortic

Cite this article as: Zschaler S, Schmidt G, Kukucka M, Syrmas G, Zaschke L, Kurz SD. How to prevent inadvertent emergency anticoagulation in acute type A aortic dissection: when in doubt, don't. Cardiovasc Diagn Ther 2018;8(6):805-810. doi: $10.21037 /$ cdt.2018.10.13
Dissection in Emergency Department Patients is Rare. West J Emerg Med 2015;16:629-31.

22. Olsson C, Thelin S, Stahle E, et al. Thoracic aortic aneurysm and dissection. Increasing prevalence and improved outcomes reported in a nationwide populationbased study of more than 14000 cases from 1987-2002. Circulation 2006;114:2611-8

23. Hagan PG, Nienaber CA, Isselbacher EM, et al. The International Registry of Acute Aortic Dissection (IRAD): new insights into an old disease. JAMA 2000;283:897-903.

24. Graber M, Gordon R, Franklin N. Reducing Diagnostic Errors in Medicine: What's the Goal? Acad Med 2002;77:981-92.

25. Graber M. Crushing Chest Pain: A Missed Opportunity. Available online: https://psnet.ahrq.gov/webmm/case/45/ crushing-chest-pain-a-missed-opportunity

26. Fatima S, Sharma K. Painless Aortic Dissection Diagnostic Dilemma With Fatal Outcomes: What Do We Learn? J Investig Med High Impact Case Rep 2017;5:2324709617721252.

27. Nadour W, Goldwasser B, Biederman RW, et al. Silent aortic dissection presenting as transient locked-in syndrome. Tex Heart Inst J 2008;35:359-61.

28. Croskerry P. The importance of cognitive errors in diagnosis and strategies to minimize them. Acad Med 2003;78:775-80.

29. Harvey AM, Bordley J, Barondess JA. Differential Diagnosis: The Interpretation of Clinical Evidence (3rd Edition). Philadelphia: W.B. Saunders Co., 1979.

30. Hemmert K, Macias M. Aortic Dissection: Practice Update. Available online: http://nuemblog.com/blog/ aortic-dissection 